\title{
Combining Machine Learning with Bayesian Inverse Modelling to Estimate the Conditional Probability of Developing Oropharyngeal Cancer following an Oral Human Papilloma Virus Infection
}

\author{
*Prerna Tewari ${ }^{1,2}$, *Eugene Kashdan ${ }^{3}$, Cathal Walsh ${ }^{4}$, Cara M Martin ${ }^{1,2}$ Andrew C Parnell ${ }^{5}$, John J \\ O'Leary ${ }^{1,2}$ \\ ${ }^{1}$ Dept. Histopathology and Morbid Anatomy, Trinity College Dublin, Dublin, Ireland \\ ${ }^{2}$ Dept. Pathology, Coombe Women \& Infants University Hospital, Dublin, Ireland \\ ${ }^{3}$ College of Business, University College Dublin, Dublin, Ireland \\ ${ }^{4}$ Dept. Mathematics and Statistics, University of Limerick, Limerick, Ireland \\ ${ }^{5}$ Hamilton Institute, Insight Centre for Data Analytics, Maynooth University, Kildare, Ireland \\ *Equal contribution
}

\section{Corresponding authors:}

Prerna Tewari

Dept. Histopathology and Morbid Anatomy,

Trinity College Dublin/Coombe Women and Infants University Hospital,

Dublin 8, Ireland

Email: tewarip@tcd.ie

Eugene Kashdan

College of Business

University College Dublin

Dublin 4, Ireland

Email: eugene.kashdan@ucd.ie

Keywords: Human Papillomavirus, Oropharyngeal cancer, Machine learning 
Abstract

\section{Background}

3 Despite an epidemic increase in the prevalence of Human Papilloma Virus (HPV) related Oropharyngeal

4 Squamous Cell Carcinomas (OPSCCs) in Northern America and parts of Europe, there is virtually no

5 information about the natural history of these cancers. The lack of well-defined precursor lesions and

6 limited data on oral HPV persistence and clearance rates, poses a challenge for disease modelling. We

7 propose a novel mathematical modelling approach to estimate the conditional probability of developing

8 HPV related OPSCCs following a prevalent HPV infection and other covariates.

\section{Methods}

10 We developed a double-Bayes method, whereby a Bayesian machine learning model first estimates the 11 probability of an individual having an oral HPV infection, given OPSCC and other covariate information.

12 The model is then inverted using Bayes' theorem to reverse the probability relationship. The mathematical

13 model was derived from two datasets representing the adult population in the United States (US), the

14 Surveillance Epidemiology and End Results Program (SEER) Head and Neck with HPV Status Database

15 and the National Health and Nutrition Examination Survey (NHANES) 2011-2014.

\section{Results}

17 The model dataset contains 8,623 subjects of which $70.7 \%$ had a prevalent oral HPV positive infection.

18 When stratified by age, sex, marital status and race/ethnicity, the model estimated higher conditional

19 probability for developing OPSCCs following an oral HPV infection in non-Hispanic White males and

20 females compared to other race/ethnicities. Non-Hispanic White males with an oral HPV infection had

21 nearly two fold higher risk of developing OPSCC than non-Hispanic White females (10.6 cases per 10,000

22 thousand vs 5.05 cases per 10,000) in the age range 50-60. 


\section{Conclusion}

24 We have employed a novel statistical approach to estimate the conditional probability of developing

25 OPSCCs following an oral HPV infection and covariates age, sex, ethnicity and marital status in the US

26 population. We recognise that at best this is a first guess estimate of a natural history model of HPV driven

27 OPSCCs within the existing limitations of the model.

28

29 


\section{Background}

31 HPV infections are now firmly established as the primary cause of cervical cancers, some anogenital

32 cancers, and a subset of Head and Neck cancers [1-3]. Head and neck cancers represent a diverse group of

33 cancers that develop from different anatomical sites; oral cavity, oropharynx (tonsils, base of tongue), nasal

34 cavity, nasopharynx, hypopharynx and larynx [4]. While there has been a decrease in the incidence of

35 laryngeal, hypopharyngeal and oral cavity cancers over the last few decades, due to decrease in smoking

36 rates, a sharp increase in the incidence of oropharyngeal squamous cell carcinomas (OPSCCs) has been

37 observed over the same period in several developed countries worldwide [5]. The increase in OPSCC

38 incidence rates has been causally linked to the growing prevalence of HPV infections presumably acquired

39 via increased oral exposure to infected anogenital sites with changing sexual behaviour [6].

40 In the United States (US), the annual incidence rate of OPSCCs has now surpassed that of cervical cancer,

41 with similar trends projected for other developed countries, there is a growing concern for managing disease

42 burden [7]. While cervical cancer has been managed effectively by well-established public health measures

43 including screening and prophylactic HPV vaccination, similar efforts have yet to be established for

44 OPSCCs $[8,9]$.

45 Unlike cervical cancer, precursor lesions are not associated with OPSCCs, but it is likely that a subclinical

46 HPV infection that persists for decades precedes the development of these cancers similar to cervical cancer.

47 The key parameters that govern the natural history of oral HPV infection (transition from infection to

48 malignancy) remain largely ill-defined because they cannot be easily inferred from experimental data.

49 While several population based studies have reported oral HPV infections prevalence rates at 4-7.0\% in

50 healthy populations with similar associated risk factors as the ones reported for OPSCCs including gender,

51 sexual behaviour and current tobacco use, limited data exists on oral HPV persistence and clearance rates

52 and associated risk factors $[10,11]$.

53 Unravelling the trajectory of oral HPV infections and associated risk factors that promote malignant

54 transformation is crucial to the development of successful primary and secondary prevention strategies. 
55 Mathematical models have been previously been used successfully to estimate some of these ill-defined

56 parameters in cervical cancer [12]. A comprehensive mathematical model incorporating data on oral HPV

57 prevalence, persistence and/or clearance rates as well as demographics, lifestyle risk factors and sexual

58 history will help to delineate the natural history of HPV in OPSCCs and associated risk factors.

59 Our aim is to estimate the conditional probability of developing OPSCCs following an oral HPV infection

60 along with socio-demographic and other covariate information. To achieve this, we developed a novel

61 modelling approach, which calibrates a machine learning method using inverse conditional probabilities.

62 We made use of the Bayesian Additive Regression Trees (BART) machine learning approach as it allows

63 for complex non-linear interactions between variables and produces probabilistic confidence and prediction

64 intervals. We stress that our approach is a proof-of-concept and outline some possible extensions.

\section{Materials and Methods}

\section{Datasets}

67 The first dataset is from the Surveillance, Epidemiology, and End Results (SEER) program which provides

68 information on cancer statistics in the U.S. population [13]. Since 2010, SEER has collected data on the

69 oral HPV status of Head and Neck Cancer patients https://seer.cancer.gov/seerstat/databases/hpv. Data is

70 available for patients diagnosed between 2010 and 2016 in 12 SEER registries. The oral HPV status

71 information has been recoded as: 1) HPV Negative; 2) HPV Positive; 3) Unknown/NA. The data also

72 includes individual-level and aggregated county-level demographic and socio-economic information.

73 The second dataset we use for the analysis was provided by the National Health and Nutrition Examination

74 Survey (NHANES) http://www.cdc.gov/nchs/nhanes.htm. NHANES is a stratified, multistage, clustered

75 probability sample that is representative of the noninstitutionalized, civilian US population [14]. NHANES

76 includes a series of surveys as well as laboratory tests and detailed questionnaires. 
77 Since 2011, NHANES has been collecting data on oral HPV infections in the US population. Participants

78 provided a 30-second oral rinse and gargle with mouthwash. DNA from oral exfoliated cells was evaluated

79 by polymerase chain reaction and type-specific hybridization for HPV detection.

\section{Data Wrangling and Exploratory Data Analysis}

81 The oral HPV data and demographic characteristics of the Head and Neck cancer patients was extracted

82 from the specialised "Head and Neck with HPV Status" SEER database (access granted on request). Only

83 those cases that were classified as oropharynx based on ICD-O-3 site codes with a confirmed oral HPV

84 status were considered for data modelling.

85 The NHANES data is stored in SAS format as a separate file for each group of variables and each period

86 of the survey. The datasets for 2011-2012 and 2013-2014 surveys provided the most granular demographic

87 data and were combined for analysis. The files were imported into $\mathrm{R}$ and linked through the unique

88 respondent ID. The data for minors (14-17 years old) is restricted and not considered for the analysis.

89 Since, we propose to combine the SEER and NHANES datasets together in a Bayesian probability

90 calculation, we require them to have variables which can be matched together. Unfortunately, this

91 necessitates removing all variables which cannot be similarly coded. The variables that remain after

92 recoding are: age, sex, marital status, and race/ethnicity (non-Hispanic Blacks, non-Hispanic Whites,

93 Hispanics and other races including American Indian/Alaska Native, Asians and Pacific Islanders).

\section{Data Modelling}

95 To estimate the conditional probability of developing OPSCCs following an oral HPV infection, we use a

96 novel double-Bayesian method whereby a Bayesian machine learning model is first used to estimate the

97 probability of an individual having an oral HPV infection, given OPSCC and other covariate information

98 from the SEER data. This model is then inverted using Bayes' theorem to reverse the probability

99 relationship. The inversion involves corrections using incidence values which are obtained from both SEER

100 and NHANES data. We have made our code available at www.github.com/andrewcparnell/HNSCC for 
101 those wishing to confirm or extend our analysis. However we note that the SEER oral HPV data are

102 restricted and not included in the Github repository.

\section{Notation}

104 We define $y$ as the event that an individual has OPSCC and $z$ as the event that an individual has an oral

105 HPV infection. We define $x_{j}$ to be the covariate values for an individual on covariate $j$, with $j=1, \ldots, M$

106 covariates, and write $x$ to be the set of all covariates for an individual. In practice $M=4$ with $x_{j}$

107 representing, respectively, age, sex, marital status and race/ethnicity.

\section{Overarching Bayesian Framework}

109 We use Bayes' theorem to invert the probabilities and obtain our desired goal $P(y \mid z, x)$ for an individual.

110 That is, the probability that an individual has OPSCC given that they have an oral HPV infection and

111 covariate values $x$. To estimate $P(y \mid z, x)$ directly we would require a longitudinal data set for which all

112 individuals had an oral HPV infection and only a subset developed OPSCC.

113 We thus calculate:

$$
P(y \mid z, x)=\frac{P(z \mid y, x) P(y \mid x)}{P(z \mid x)}
$$

115 where now $P(z \mid y, x)$ is the probability of an individual getting HPV given they have OPSCC, $\operatorname{Pr}(y \mid x)$ is

116 the probability of getting OPSCC given covariates $x$, and $\operatorname{Pr}(z \mid x)$ is the probability of getting HPV given

117 covariates $x$. We discuss how we obtain numerical estimates for each of these in turn below but, briefly,

$118 P(z \mid y, x)$ is obtained from the SEER data via our BART model, $\operatorname{Pr}(y \mid x)$ is obtained from SEER incidence 119 estimates, and $\operatorname{Pr}(z \mid x)$ is obtained from NHANES incidence estimates of oral HPV.

\section{BART}

121 The predictive probabilities $P(z \mid y, x)$ were calculated from the SEER data by employing BART. We use 122 the relationship: 


$$
\operatorname{Pr}(z \mid y, x)=\int p(z, \Theta \mid y, x, D) \partial \Theta
$$

124 where $\Theta$ are a set of parameters (detailed below) arising from posterior distribution calculated by the BART 125 model, and $D$ is the SEER data set consisting of triples $\left(x_{i}, y_{i}, z_{i}\right)$ for individuals $i=1, \ldots, 8623$.

126 The probability density $p(z, \Theta \mid y, x, D)$ is created from:

$$
p(z, \Theta \mid y, x, D)=p(\Theta \mid D) p(z \mid \Theta, y, x, D) \quad \text { (iii) }
$$

128 where $p(\Theta \mid D)$ is the BART posterior distribution and $p(z \mid \Theta, y, x, D)$ is the predictive distribution of oral 129 HPV for a new individual with OPSCC status $y$ and covariate value $x$.

130 The BART posterior distribution is built on a relationship that estimates the probability of oral HPV 131 occurrence from OPSCC and covariates via a latent probit model [15], where first:

$$
p(\Theta \mid D) \propto p(\Theta) \prod_{i=1}^{8623} p\left(y_{i} \mid x_{i}, z_{i}, \Theta\right)
$$

133 where $p(\Theta)$ is a prior distribution on a set of parameters $\Theta$ and $\prod p\left(y_{i} \mid x_{i}, z_{i}, \Theta\right)$ is a likelihood term. In a 134 BART probit classification model the likelihood is structured so that, at the top level:

$135 y_{i}=\left\{\begin{array}{c}1 \text { if } \widetilde{y}_{i}>0 \\ 0 \text { otherwise }\end{array}\right.$ where $\widetilde{y}$ is a latent parameter which is given a normally distributed likelihood:

$$
\tilde{y}_{i} \sim N\left(\mu_{i}, 1\right)
$$

138 The key component of BART is that the $\mu_{i}$ parameters are set to be a sum of regression trees, i.e.:

$$
\mu_{i}=\sum_{j=1}^{J} g_{j}\left(x_{i}, z_{i}, \theta_{j}\right)
$$

140 with $\theta_{j}$ controlling the leaf node parameters of tree $j$. Each tree is made up of a set of decision nodes (based 141 on $x_{i}$ and $z_{i}$ ) to provide a prediction $\theta_{j}$ which, when summed across trees, produces an estimate of $\widetilde{y}_{i}$ which 142 is then compared with $y_{i}$ through the equation above. The full set of parameters $\Theta$ contains all the tree 
143 decision parameters $g_{j}$, the prediction parameters $\theta_{j}$ and the latent normal parameters $\widetilde{\mu}_{i}$ for all

144 observations.

145 The model fitting stage of BART involves first guessing at values of the parameters and updating them

146 using Markov Chain Monte Carlo [16] to produce a large set of estimates of the parameters. It can be shown

147 that the latent parameter formulation above can be re-expressed as a binomial probit model with:

$$
y_{i} \mid x_{i}, z_{i} \sim \operatorname{Bin}\left(1, \phi\left(\mu_{i}\right)\right)
$$

149 where $\phi$ is the standard normal cdf. We use the posterior estimates of $\phi(\mu)$ as probabilities of oral HPV 150 given OPSCC.

151 We run the probit BART model using the bartMachine package, which by default uses $J=50$ trees and 152 produces 1000 posterior samples of the parameters [17].

\section{Bayesian Inversion with BART}

154 Our approach, taken together, involves the following steps:

155 1. Fit the BART probit model in equation (vi) to the SEER data set to estimate the probability of an 156 oral HPV infection given OPSCC and covariates for any given adult.

1572 2. For each desired new set of values $x$ comprising a set of covariate values:

a. Simulate from the posterior distribution a predicted probability of oral HPV given OPSCC for these covariate values.

b. Look up the SEER and NHANES incidence rates for that combination of covariates $x$. These are assumed known without uncertainty. 
c. Compute the probability of OPSCC given oral HPV and covariates using equation (i). This involves multiplying the simulated probability from step (a) above by the ratio of the incidence rates.

3. Repeat step 2 to form a posterior distribution of probabilities.

166 We subsequently summarise these probabilities to produce $95 \%$ posterior uncertainty intervals.

\section{Results}

\section{Study Population}

169 The model dataset contains 8,623 individuals with a mean age of 60.9 years. Males accounted for $82.9 \%$ of 170 the population with an oral HPV infection rate of $73.6 \%$. Females constituted $17.1 \%$ of the entire population

171 and had an oral HPV infection rate of 56.4\%. Amongst the different race/ethnic groups, non-Hispanic

172 Whites represented $80.1 \%$ of the entire dataset with an oral HPV prevalence rate of $74.4 \%$. Characteristics

173 of the study participants included in the model are presented in Table 1.

174 The NHNAES study population included 9,134 respondents with a confirmed oral HPV data. The mean

175 age of participants was 42.1 years. Males comprised $49.2 \%$ of the population with an oral HPV prevalence

176 rate of $11.9 \%$ and females represented $50.8 \%$ with an oral HPV prevalence rate of $3.8 \%$. Demographic

177 details and oral HPV prevalence rates are summarised in Table 2.

\section{Performance of the BART Model}

179 The BART model was run on the SEER HPV data using the default 50 trees and 1000 posterior iterations

180 (removing a proportion for a warm-up period). We use $75 \%$ of the data for training purposes and the

181 remaining $25 \%$ constituted the test data set with similar proportions of HPV negative/positive as the full 182 dataset.

183 We evaluated the performance of the model via the Receiver Operator Characteristic (ROC) curve and the

184 Area Under the Curve (AUC) value. Using only age, sex, marital status and race/ethnicity as covariates, we 
185 obtained an AUC value of 0.70 . We also had the possibility of using further covariates such as smoking 186 and employment status but these are only available at an aggregated level; no model we tried increased the 187 AUC beyond 0.70. In the end we removed these extra covariates for simplicity in the final interpretation of 188 the model.

189 Conditional Probability of Developing OPSCC given Oral HPV by Age, Sex, Marital Status and 190 Race/Ethnicity

191 The estimated probabilities of an individual developing OPSCC following an oral HPV infection are shown

192 in Figure 1. We note that due to the nature of the machine learning model, all probabilities are calculated 193 on the full set of covariates: age, sex, marital status and race/ethnicity.

194 The risk estimates for males show an increased probability for non-Hispanic White males up to the age of 19560 (10.6 cases per 10,000), with lower risk estimates for Hispanics (5.6 cases per 10, 0000) and non196 Hispanic Blacks (4.6 per 10,000). The risk estimates for females show similar trends with an increased

197 probability for non-Hispanic White females (4.95 cases per 10,000) of developing OPSCCs, whilst risk

198 estimates for Non-Hispanic Blacks and Hispanics are broadly similar for the age range 40-50. It appears 199 that Hispanic females have a lower chance of developing OPSCC than other ethnicities, but there is likely 200 considerable uncertainty in these values due to lower sample sizes.

201 Conditional Probability of Developing OPSCC given HPV by Age, Sex and Marital Status for Non202 Hispanic Whites

203 The estimated probabilities of an individual developing OPSCC given they have HPV for married Non204 Hispanic Whites by age is shown in Figure 2. Whilst there is a clear increase across ages for both sexes, 205 there appears to be little difference in the probabilities of developing OPSCCs for non-Hispanic White 206 males and females up to the age of 50. For the age range 50-60, males have a substantially higher risk of 207 developing OPSCCs than females (10.6 cases per 10,000 vs 5.05 cases per 10,000) following an HPV 
208 infection. However, we observe a substantial increase in the number of cases for women >60 and a decline

209 in the number of OPSCC cases in males aged $>60$ and above.

\section{Discussion}

211 We have developed a novel mathematical modelling approach - a "double Bayes" that inverts the results

212 of a machine learning BART method using Bayes Theorem to estimate the correct conditional probabilities

213 for developing OPSCCs following a prevalent oral HPV infection and other associated covariates: age, sex,

214 marital status and race/ethnicity in the US population. This approach represents the first step in delineating

215 the natural history of HPV related OPSCCs, a disease notoriously difficult to model given the lack of well-

216 defined histological end-points and associated HPV infections.

217 The model estimates a substantially higher risk of HPV related OPSCCs for married non-Hispanic White

218 males and females when compared to other races/ethnic groups. We appreciate that there is but likely

219 considerable uncertainty in the probability estimates due to lower sample sizes, nonetheless similar risk

220 estimates have been observed in previous cohort studies on HPV related OPSCCs, although with the caveat

221 that the risk estimates we report are derived from Bayesian modelling [5, 18].

222 Despite the mathematical novelty of our approach, and in particular the ability to invert the association

223 between OPSCC and HPV, we foresee several difficulties and issues with the results we present. The first

224 is that we have no means to estimate the time interval between an individual having an oral HPV infection

225 and subsequent development of OPSCC as no longitudinal follow-up data was available in either of the

226 datasets. The NHANES dataset is a cross-sectional dataset with a single time point measure of oral HPV

227 infections in healthy individuals. The lack of longitudinal follow-up data prevents estimation of oral HPV

228 persistence, clearance rates and the potential role in malignant transformation. While the SEER dataset

229 provides no data on oral HPV infection in individuals before the diagnosis of OPSCC. An ideal data set

230 would collect both time of diagnosis of oral HPV and OPSCC, and use a double censoring model to estimate

231 the time lag similar to HIV natural history models [19]. A second issue relates to the uncertainty estimates 
232 for the incidence of OPSCC or HPV. If these were available, we could propagate them through our inversion

233 technique to produce more conservative uncertainty intervals. Currently we believe our results, which we

234 stand over as a best first guess, underestimate the interval widths at any given confidence level.

235 A third limitation is the cross-categorisation of various covariates collected as part of the analysis. Between

236 SEER and NHANES, there are different levels of categorisation of both age and race/ethnicity which

237 requires some level of re-coding and so weakens the final probabilistic predictions we create. Furthermore,

238 due to limited /lack of data on smoking, sexual behaviour and sexual history on individuals in the SEER

239 dataset, we were unable to include these known risk factors for HPV related OPSCCs as covariates in our

240 model [20].

241 The major strength of the proposed modelling approach lies in developing and validating the oral HPV

242 natural history model in a large sample size of participants, pooled through two well characterised

243 datasets; NHANES and SEER, representing healthy individuals and OPSCC patients derived from the

244 same population.

\section{Conclusion}

246 In summary, we have developed and described a novel statistical modelling approach to estimate the

247 conditional probability of developing OPSCCs following an HPV infection and covariates age, sex, marital

248 status and race/ethnicity in the US population. We appreciate the limitations of the proposed model and at

249 best recognise that this is a first guess estimate of a natural history model of HPV driven OPSCCs.

250 Abbreviations:

251 HPV: Human Papillomavirus

252 HNSCC: Head and Neck Squamous Cell Carcinoma

253 OPSCC: Oropharyngeal Squamous Cell Carcinoma 
254 BART: Bayesian Additive Regression Trees

255 NHANES: National Health and Nutrition Examination Survey

256 SEER: Surveillance Epidemiology and End Results Program

257 AUC: Area under the curve

258 ROC: Receiver operating characteristic

259 Author contributions: JOL, CM, PT, EK and ACP designed the study. EK and PT identified datasets and

260 extracted data for model development. EK, ACP and CW developed the mathematical modelling approach.

261 EK, PT and ACP drafted the manuscript. All authors edited, revised and approved the final manuscript for 262 submission.

263 Funding: Health Research Board, Ireland. Grant/Award Numbers ICE-2015-1037.

264 Availability of data and materials: Code available at www.github.com/andrewcparnell/HNSCC. The

265 SEER HPV data are restricted and are not included in the Github repository.

266 Ethics approval and consent to participate: Not applicable

267 Consent for publication: Not applicable

268 Competing interests: Not applicable

269 Acknowledgements

270 We are grateful to the SEER HPV database for providing access to the restricted HPV data.

271 EK and ACP were paid for a portion of this work through HRB award ICE-2015-1037.

272 ACP is funded by Science Foundation Ireland Career Development Award grant 17/CDA/4695 and a SFI 273 centre grant 12/RC/2289_P2 


\section{References}

275 1. Walboomers JM, Jacobs MV, Manos MM, Bosch FX, Kummer JA, Shah KV, et al. Human

276 papillomavirus is a necessary cause of invasive cervical cancer worldwide. J Pathol. 1999; 189(1):12-9.

277 2. Koutsky L. Epidemiology of genital human papillomavirus infection. Am J Med. 1997; 102: 3-8.

278 3. Gillison ML, Koch WM, Capone RB, Spafford M, Westra WH, Wu L et al. Evidence for a causal 279 association between human papillomavirus and a subset of head and neck cancers. J Natl Cancer Inst. 2000; $28092: 709-720$.

281 4. Cognetti DM, Weber RS, Lai SY. Head and neck cancer: an evolving treatment paradigm. Cancer. 2008; 282113 (7 Suppl):1911-32.

283 5. Chaturvedi AK, Engels EA, Anderson WF, Gillison ML. Incidence trends for human papillomavirus284 related and -unrelated oral squamous cell carcinomas in the United States. J Clin Oncol 2008; 26:612-9.

285 6. Dahlstrom KR, Li G, Tortolero-Luna G, Wei Q, Sturgis EM. Differences in history of sexual behavior 286 between patients with oropharyngeal squamous cell carcinoma and patients with squamous cell carcinoma 287 at other head and neck sites. Head Neck. 2011; 33(6):847-55.

288 7. Jemal A, Simard EP, Dorell C, Noone AM, Markowitz LE, Kohler B, et al. Annual Report to the Nation 289 on the Status of Cancer, 1975-2009, featuring the burden and trends in human papillomavirus (HPV)290 associated cancers and HPV vaccination coverage levels. J Natl Cancer Inst. 2013;105(3):175-201.

291 8. Crosbie EJ, Einstein MH, Franceschi S, Kitchener HC. Human papillomavirus and cervical cancer. 292 Lancet. 2013; 382(9895):889-99.

293 9. Huang SH, O'Sullivan B, Waldron J. The Current State of Biological and Clinical Implications of Human 294 Papillomavirus-Related Oropharyngeal Cancer. Semin Radiat Oncol. 2018; 28(1):17-26. 
295 10. Gillison ML, Broutian T, Pickard RK, Tong ZY, Xiao W, Kahle L, et al. Prevalence of oral HPV 296 infection in the United States, 2009-2010. JAMA. 2012; 307(7):693-703.

297 11. Kreimer AR, Pierce Campbell CM, Lin HY, Fulp W, Papenfuss MR, Abrahamsen M, et al. Incidence 298 and clearance of oral human papillomavirus infection in men: the HIM cohort study. Lancet. 2013; 299 382(9895):877-87.

300 12. Kim JJ, Kuntz KM, Stout NK, Mahmud S, Villa LL, Franco EL, et al. Multiparameter calibration of a 301 natural history model of cervical cancer. Am J Epidemiol. 2007; 166(2):137-50.

302 13. Surveillance, Epidemiology, and End Results (SEER) Program (www.seer.cancer.gov) SEER*Stat

303 Database: Incidence - SEER 18 Regs Custom Data Head and Neck (select schemas with HPV recode and 304 additional treatment fields), Nov 2017 Sub (2013-2015) - Linked To County Attributes - Total U.S., 1969-

3052016 Counties, National Cancer Institute, DCCPS, Surveillance Research Program, released April 2018, 306 based on the November 2017 submission.

307 14. Sanders AE, Slade GD, Patton LL. National prevalence of oral HPV infection and related risk factors 308 in the U.S. adult population. Oral Dis. 2012; 18(5):430-41.

309 15. Chipman, Hugh A., Edward I. George, and Robert E. McCulloch. 2010. "BART: Bayesian Additive 310 Regression Trees.” Ann. Appl. Stat. 4 (1). The Institute of Mathematical Statistics: 266-98.

311 16. Flegal, J. M. and Jones, G. L. (2011). Implementing MCMC: estimating with confidence in Brooks, S., 312 Gelman, A., Jones, G. L., and Meng, X. L., editors, Handbook of Markov116chain Monte Carlo, pages 313 175-197. Chapman \& Hall / CRC, Boca Raton, Florida.

314 17. Kapelner, Adam, and Justin Bleich. bartMachine: Machine Learning with Bayesian Additive 315 Regression Trees. Journal of Statistical Software 2014; 70 (4): 1-40.

316 18. Logan, Brent R, Rodney Sparapani, Robert E McCulloch, and Purushottam W Laud. Decision Making 317 and Uncertainty Quantification for Individualized Treatments Using Bayesian Additive Regression Trees. 
318 Statistical Methods in Medical Research 2017; SAGE Publications Sage UK: London, England, 3190962280217746191.

320 19. Lam, L. The analysis of doubly censored survival data. An application to data collected from the 321 Amsterdam cohort studies on HIV infection and AIDS. Technical Report WBBM Report Series 36, Delft 322 University of Technology, Delft, The Netherlands 1997.

323 20. Jiang S, Dong Y. Human papillomavirus and oral squamous cell carcinoma: A review of HPV-positive 324 oral squamous cell carcinoma and possible strategies for future. Curr Probl Cancer. 2017; 41(5):323-327. 